\title{
Efficient Job Recommender System
}

\author{
Sheela Bankar ${ }^{1}$, Ambesh Vishwakarma ${ }^{2}$, Siddhi Sarolkar ${ }^{3}$ \\ Assistant Professor, IT Dept, ISB\&M SOT, Pune, India ${ }^{1}$ \\ Student, IT Dept, ISB\&M SOT, Pune, India ${ }^{2,3}$
}

\begin{abstract}
Open source resume is web application to help programming advancement groups to enroll designers in view of their open source framework commitment exercises. This instrument gives bits of knowledge into designers' Practical commitments to open source framework ventures, which can be valuable criteria for judging whether they are appropriate for joining the advancement group in view of their diverse capabilities. To beat the Empowerment issues many employment entrances appeared despite the fact that these entries are attempting their best we are confronting numerous challenges to get reaction from the corporate side, in the meantime even corporate human resource individuals are confronting troubles to discover the representative who fits their necessity. Activity is expected to conquer these many difficulties and make everything less demanding for the clients.
\end{abstract}

Keywords: Open Source Software; Mining Software Repositories; Developer Expertise; Visualization Tool.

\section{INTRODUCTION}

The genuine issue is to make a website for the candidates, is produced for making an intelligent occupation opportunity for candidates. This web application is to be imagined in its present shape as a dynamic website requiring steady updates both from the searchers and additionally the organizations. Overall the target of the venture is to empower jobseekers to put their resumes and organizations to distribute their opportunities. It empowers jobseekers to post their resume, scan for occupations, see individual employment postings.

It will give different organizations to put their opening profile on the site and furthermore have an alternative to pursuit competitor resumes. Aside from this there will be a trough module for the possibility to roll out improvements to the database content. It comprises of 5 modules: The clients of this framework are scanning for employment, enrollment their personal, educational, aptitudes, venture and resume subtle elements. This framework is composed such a route, to the point that the clients can undoubtedly interface with the framework with least information to program the net and organization rules.

\section{RELATED WORK}

In this segment, we acquaint earlier business related with this paper. Social Network Service(SNS) has been a standout amongst the most well known themes far and wide since the most recent decade [1]. As a After effect of the quick increment in SNS clients, a few reviews connected information mining methods to analyze SNS for investigating drifting subjects and looking for attractive group for oneself [7].

GitHub, as the biggest OSS engineers' group, is also investigated and analyzed for the motivations behind visualizing OSS group or recognizing OSS supporters. In late year, numerous associations give careful consideration to GitHub information analysis [1].

Gousios et al. gathered fundamentally vast sum of information from GitHub by utilizing official API and such information was continued to shape a venture, named as GHTorrent, to exhibit the dataset of open activities in GitHub [4]. Hauff et al. Proposed the pipeline for automatically coordinating between employment notices and designers on GitHub [5]. Nonetheless, they gathered designer's profile through README, in which the concerned information for our review is not really included. (e.g., the area designer lives or year of programming knowledge). Subsequently, we concentrate on utilizing GitHub API to gather profile information.

\section{IMPLEMENTATION}

There are 2 major module in our application i.e. Candidate module, Recruiter module etc. Candidate can register to application by filling all details in registration form. Candidate will get login credentials after registration process is finished. Similarly, login and registration of recruiter is provided. Registration and login of candidate is as shown below fig 1(a) and 1(b). 


\section{IJARCCE}

International Journal of Advanced Research in Computer and Communication Engineering ISO 3297:2007 Certified

Vol. 6, Issue 5, May 2017

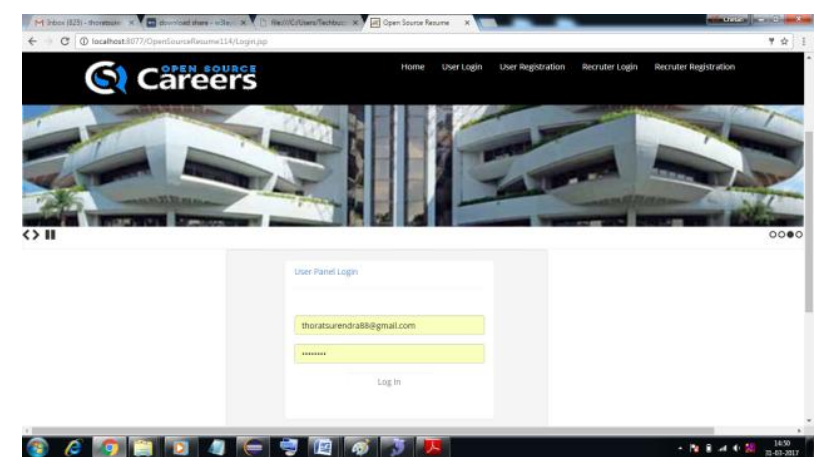

Fig 1(a)

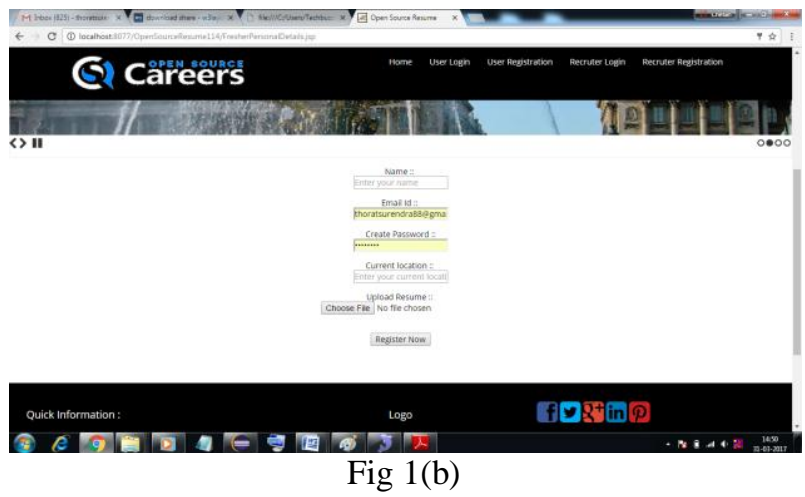

After login, candidate can view available jobs and search jobs according to their requirements. While searching jobs, candidate need to enter job title and work experienced as shown in fig 2(a)and (b). Candidate can update their profile.

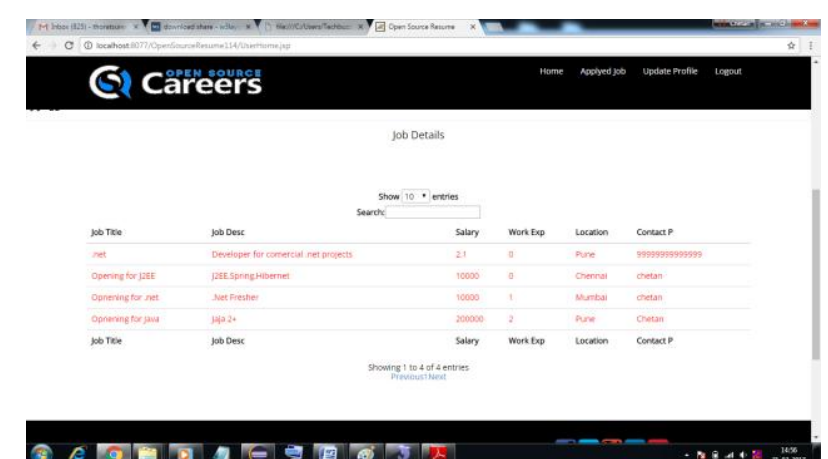

Fig 2(a)
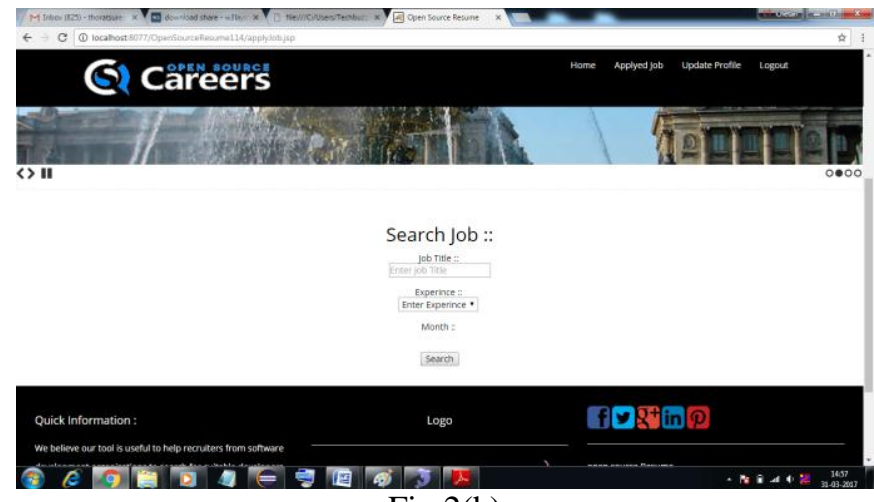

Fig 2(b)

Recruiter can post job, view applied jobs. Recruiter view uploaded job history. Recruiter can post job by entering all details as shown in fig 3(a) and 3(b). 


\section{IJARCCE}

International Journal of Advanced Research in Computer and Communication Engineering ISO 3297:2007 Certified

Vol. 6, Issue 5, May 2017

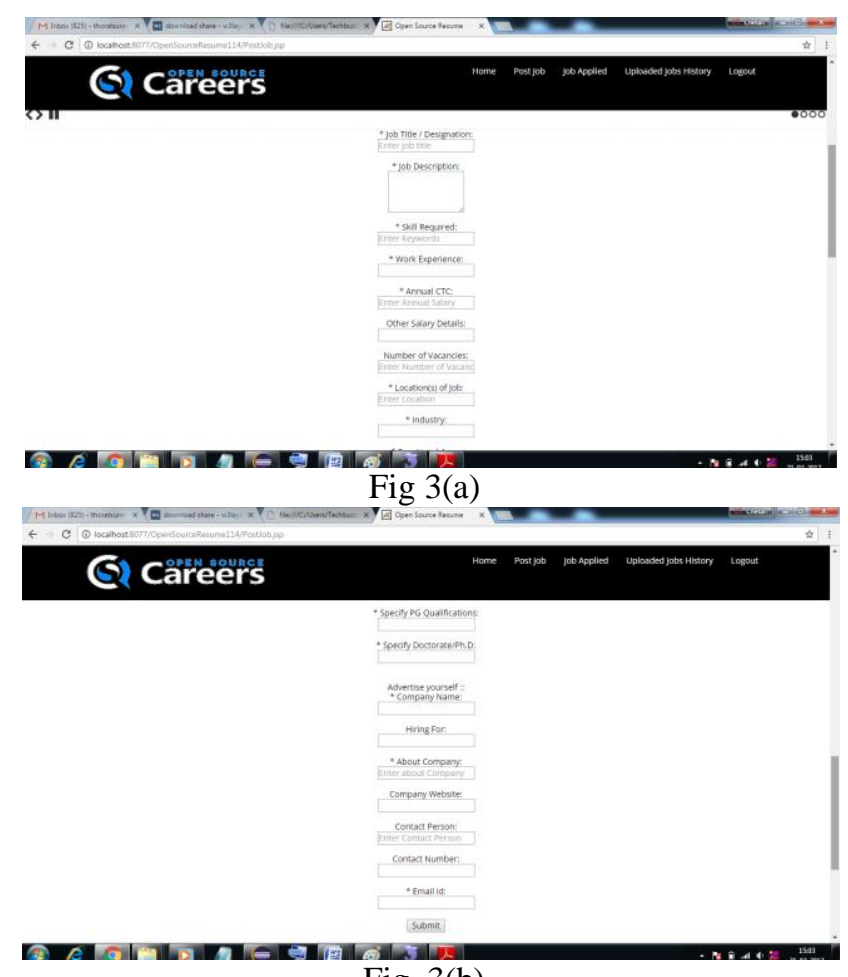

Fig. 3(b)

Recruiter can view job applied by candidate as shown fig 4 .

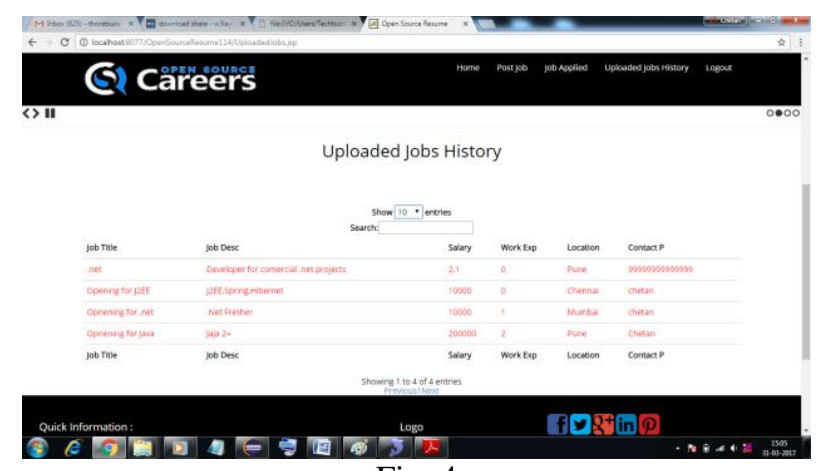

Fig. 4

\section{RESULT ANALYSIS}

We have analyzed our application by registering some candidate to this portal. Candidate did registration to our portal and used this application. We compare our proposed system with existing system and represented in graphical form.

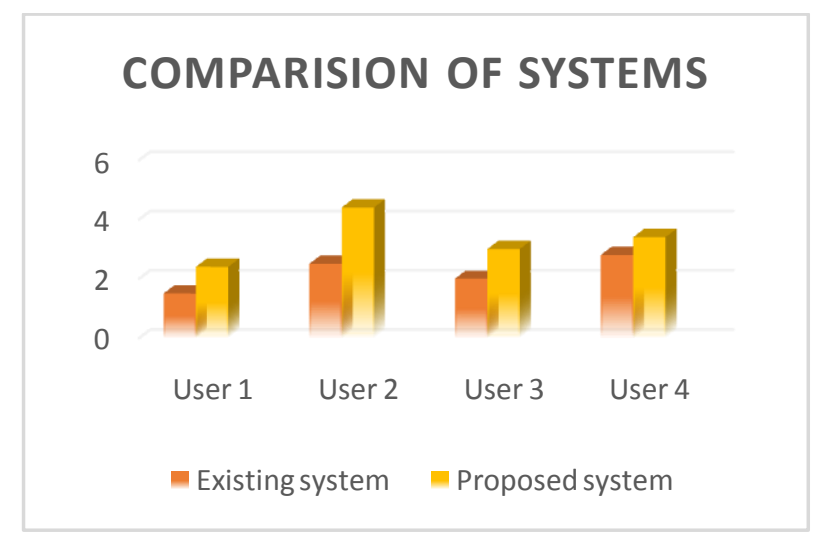




\section{CONCLUSION}

We introduced cutting job recommendation suggestion and in addition, a near review for its methodologies that proposed by literary works. Moreover, we checked on regular recommender framework strategies and the enrolling procedure related issues. We infer that the field of occupation suggestions is as yet unripe and require encourage enhancements

\section{REFERENCES}

1. S. T. Al-Otaibi and M. Ykhlef, “A survey of job recommender systems,” International Journal of the Physical Sciences, vol. 7(29), pp. 51275142, July, 2012.

2. S. T. Zheng, W. X. Hong, N. Zhang and F. Yang, "Job recommender systems: a survey," In Proceedings of the 7th International Conference on Computer Science \& Education (ICCSE 2012), pp. 920-924, Melbourne, Australia, July, 2012.

3. K. Yu, G. Guan and M. Zhou, "Resume information extraction with cascaded hybrid model," In Proceedings of the 43rd Annual Meeting of the ACL, pp. 499-506, Ann Arbor, Michigan, June, 2005.

4 I. Paparrizos, B. B. Cambazoglu and A. Gionis, "Machine learned job recommendation," In Proceedings of the fifth ACM Conference on Recommender Systems, pp. 325-328, Chicago, USA, October, 2011.

5. M. Adedoyin-Olowe, M. M. Gaber, and F. T. Stahl. A survey of data mining techniques for social media analysis. CoRR, abs/1312.4617, 2013. II

6. H. W. Ye, “A Personalized Collaborative Filtering Recommendation Using Association Rules Mining and Self-Organizing Map,” Journal of Software, vol. 6(4), pp.732-739, 2011.

7. L. Hu, W. B. Wang, F. Wang, X. L. Zhang and K. Zhao, "The Design and Implementation of Composite Collaborative Filtering Algorithm for Personalized Recommendation,” Journal of Software, vol. 7(9), pp. 2040-2045, 2012.

8. L. Pizzato, T. Rej, T. Chung, K. Yacef, I. Koprinska and J. Kay, "Reciprocal recommenders," In Proceedings of 8th Workshop on Intelligent Techniques for Web Personalization and Recommender Systems, held in conjunction with the 18th International Conference on User Modeling, Adaptation and Personalization (UMAP 2010), Hawaii, USA, June, 2010.

9. R. Burke, "Hybrid recommender systems: survey and experiments," User Modeling and User-Adapted

10. S. T. Al-Otaibi and M. Ykhlef, “A survey of job recommender systems," International Journal of the Physical Sciences, vol. 7(29), pp. 51275142, July, 2012. 